05

\title{
Влияние изохронных отжигов на поверхностные характеристики лент металлического стекла $\mathrm{Ni}_{50} \mathrm{Ti}_{50}$
}

\author{
(ㄷ П.Н. Бутенко, В.Л. Гиляров, В.Е. Корсуков, М.М. Корсукова, Б.А. Обидов \\ Физико-технический институт им. А.Ф. Иофрфе РАН, \\ 194021 Санкт-Петербург, Россия \\ e-mail: pavel.butenko@mail.ioffe.ru
}

Поступило в Редакцию 29 апреля 2019 г.

В окончательной редакции 29 апреля 2019 г.

Принято к публикации 29 июня 2019 г.

Изучена кристаллизация металлического стекла ленты NiTi эквиатомного состава методами рентгеноструктурного анализа и атомно-силовой микроскопии. Поверхность изначальной ленты, полученной методом спиннингования, содержала только кристаллиты примесных фаз. В результате воздействия отжига в течение $10 \mathrm{~min}$ при температуре $500^{\circ} \mathrm{C}$ наблюдался фазовый переход в виде упорядочивания фазы $\mathrm{Ni}_{50} \mathrm{Ti}_{50}$. При анализе геометрии поверхности ленты рассчитаны непосредственные параметры неоднородностей, шероховатости, фрактальной размерности и величин мультифрактального формализма. Установлено, что при режиме термообработки при $500^{\circ} \mathrm{C}$ в течение $10 \mathrm{~min}$ все вышеперечисленные характеристики имели локальные экстремумы. В частности, наблюдался минимум значений ширин спектров сингулярностей, что указывало на монофрактализацию системы.

Ключевые слова: металлическое стекло, поверхность, отжиг, фазовый переход, кристаллизация, статистический анализ, мультифрактальный формализм.

DOI: $10.21883 /$ JTF.2020.02.48812.177-19

\section{Введение}

В последнее время сплав памяти формы NiTi эквиатомного состава, получаемый в виде тонких лент, значительно расширил область своего применения. С развитием таких сфер, как робототехника, биоинженерия, протезирование в медицине и пр. появилась возможность применения различных микро- и наноустройств, таких как микронасосы, микроманипуляторы, микроприводы, импланты, зонды, датчики и пр. [1-6]. Как правило, ленты нитинола в подобных приложениях имеют толщину порядка 20-30 $\mu \mathrm{m}$ и изначально являются аморфными материалами $[3,7,8]$. Основной способ получения таких лент - сверхбыстрая закалка из жидкой фазы, основной метод - спиннингование [9]. Однако для того, чтобы лента обладала эффектом памяти формы, требуется перевести материал в кристаллическое состояние, только тогда станет возможным мартенситно-аустенитное превращение, которое и лежит в основе данного эффекта [10].

Процесс кристаллизации стекол до сих пор является дискуссионным [11]. Классическая теория зародышеобразования (первая стадия кристаллизации) достаточно хорошо описывает процесс качественно, однако его количественные характеристики имеют большой разброс. Связано это с инвариантностью процесса зародышеобразования (достижения критического размера зерна), которую система имеет изначально. Металлические стекла (MC), будучи структурно и химически негомогенными материалами, изначально в той или иной степени подвержены фазовому расслоению. Поскольку структура стекла на мелких масштабах плохо изучена, неясны закономерности структурообразования, следовательно, непонятна связь между неоднородностями размерами ниже $50 \mathrm{~nm}$ и зародышеобразованием. Кроме этого, такие ленты МС, будучи достаточно тонкими объектами, имеют относительно малую толщину и развитую поверхность, которая, как известно, содержит большее количество неоднородностей, дефектов и имеет свойства, отличные от свойств в объеме материала $[12,13]$. Все это существенно усложняет ситуацию и требует ее тщательного изучения. По этой причине в условиях высокой практической ценности при использовании нитинола задача комплексного (особенно количественного) исследования этого материала в виде тонких лент актуальна.

Кинетика кристаллизации МС является сложным многофакторным явлением, которое контролируют следующие процессы: атомные перестройки, зародышеобразование, рост кристаллов, появление дальнего порядка, уменьшение свободного объема [14]. Структура, получаемая при кристаллизации, является непрерывной и случайной, зерна растут в аморфной матрице до соприкосновения друг с другом [15]. На начальных стадиях кристаллизации при нагреве образуется структура аустенита, с понижением температуры структура гомогенезируется, кристаллиты приобретают схожую геометрию, имеют характер параллельных полос, что указывает на мартенситную фазу [15]. Таким образом, фазовый переход наблюдается уже в самом начале процесса кристаллизации. Принципиально начало фазового перехода при определенных условиях на поверхности образцов может 
наблюдаться при температурах порядка $300^{\circ} \mathrm{C}[16,17]$. Однако большинство исследователей наблюдали начало кристаллизации на отметке $490^{\circ} \mathrm{C}[7,8,10]$. Уже в первые $10 \mathrm{~min}$ на поверхности образцов появлялись кристаллиты, однако объем кристаллизовался позже, при этом скорость роста фронта кристаллизации составляла величину примерно $0.015 \mu \mathrm{m} / \mathrm{s}$ (см., например, [7]).

Размер зерна кристаллитов, кроме температур отжига, сильно зависит от его продолжительности. Например, в случае ранней поверхностной кристаллизации при температурах $300^{\circ} \mathrm{C}$ (в начальный момент отжига) наблюдали появление первых нанокристаллов с размерами порядка $30 \mathrm{~nm}$ [17]. При отжиге при температуре $500^{\circ} \mathrm{C}$ в течение $5 \mathrm{~min}$ размер зерна составлял уже $80 \mathrm{~nm}$. Отжиг при $600^{\circ} \mathrm{C}$ при $45 \mathrm{~min}$ давал кристаллиты размером порядка $1500 \mathrm{~nm}$, которые заполняли весь объем изучаемой площади.

\section{1. Экспериментальные и статистические методы исследования поверхности}

В качестве экспериментальных методов при изучении структуры объема и поверхности МС при кристаллизации традиционно применяют рентгеноструктурный анализ (РСА), просвечивающую электронную микроскопию (ПЭМ), атомно-силовую (АСМ) и сканирующую туннельную микроскопии (СТМ).

Последнее время получают распространение статистические методы оценки геометрии рельефа поверхности материалов, прежде всего - фрактальный анализ $(Ф А)$ и мультифрактальный формализм (МФ) [18-21]. Используя эти подходы, можно получать достаточно полную картину трансформации поверхности при различных внешних воздействиях, которые, влияя на образец в целом, вызывают изменения в различных слоях материала, особенно в поверхностных. Реакция поверхности может описывать изменения структуры и свойств всего материала, более того, предсказывать процессы, протекающие в нем на микро- и макроуровне.

Поверхности большинства материалов являются самоафинными фракталами, поскольку направления вдоль (латеральная плоскость) и поперек (перпендикуляр к латеральной плоскости) поверхности неэквивалентны $[22,23]$. Такие поверхности обладают дробной (фрактальной) размерностью на малых масштабах, в то время как на больших масштабах размерность этих поверхностей равна двум. Более того, геометрия различных участков поверхности часто бывает неоднородной, вследствие чего фрактальная размерность (ФР или $\left.D_{f}\right)$ этих участков также различна. Такие поверхности представляют собой самоафинные мультифракталы, которые характеризуются спектром обобщенных размерностей Реньи $\left(D_{q}\right)$.

Для расчета величин $D_{f}$ и $D_{q}$ известен круг методик $[24,25]$, которые успешно применяются и дают результаты, описывающие трансформацию рельефа поверхности в зависимости: от внешних воздействий, от состояния материала; от процессов, проходящих в материале. Используя подобные методики, в определенной мере можно прогнозировать изменения в материале.

\section{2. Материалы и методика эксперимента}

Объектом исследования являлись образцы ленты МС $\mathrm{Ni}_{50} \mathrm{Ti}_{50}$, которые были получены методом спиннингования из жидкого раствора [9] на воздухе. Изучалась контактная сторона ленты (непосредственно соприкасающаяся с диском в процессе спиннингования). Ее выбор был обусловлен тем, что контактная сторона является менее однородной, в большей степени аморфизированной, содержит большее количество неоднородностей и дефектов, процессы кристаллизации и разрушения начинаются с нее $[12,13]$. Размеры образцов составляли $30 \times 5 \mathrm{~mm}$, толщина $15 \mu \mathrm{m}$. Образцы подвергались термообработке в муфельной печи (Nabertherm LE 4/11) в воздушной атмосфере по трем режимам (независимо): $400^{\circ} \mathrm{C}$ при $10 \mathrm{~min}, 500^{\circ} \mathrm{C}$ при $10 \mathrm{~min}$ и $600^{\circ} \mathrm{C}$ при $10 \mathrm{~min}$.

Наличие кристаллической составляющей и фазовый состав лент изучали методом РСА на установке ДРОН 8 (Буревестник) с использованием $\mathrm{Cu} K_{\alpha}(0.154 \mathrm{~nm})$ излучения (режим $40 \mathrm{kV}, 30 \mathrm{~mA}$ ). Поверхность образцов исследовалась с помощью метода АСМ с использованием прибора AutoProbe M5 (Park Scientific Instruments) в режиме прерывистого контакта.

По данным топограмм образцов рассчитывались статистические характеристики поверхности. Прежде всего, определялись значения среднеквадратичной шероховатости $R_{R M S}$ и реальной площади поверхности $S_{a c t}$ (не проекционная площадь исследуемого участка, а вычисленная площадь этой поверхности на конкретном масштабе). Оба этих параметра определялись с помощью стандартных методик [26].

Фрактальные характеристики поверхности (в частности $D_{f}$ ) рассчитывались с применением методики „Box counting“ для трехмерных поверхностей, в качестве элемента покрытия сетки был выбран кубический носитель. Опираясь на идею мультифрактального формализма, применялась методика расчета мультифрактальных характеристик поверхности с построением спектров сингулярностей $f(\alpha)$. Поверхность покрывалась квадратами размера $l$. В качестве мультифрактальной меры на этой решетке выбиралась высота поверхностного профиля. Вероятность заполнения квадратной ячейки определялась средней высотой профиля (сумма высот точек, принадлежащих данной ячейке, нормированная на общую сумму высот рассматриваемого участка поверхностного профиля). Статистическая сумма вычислялась стандартным способом. Из нее определялись скейлинговые экспоненты $\tau(q)$, из которых и рассчитывался 

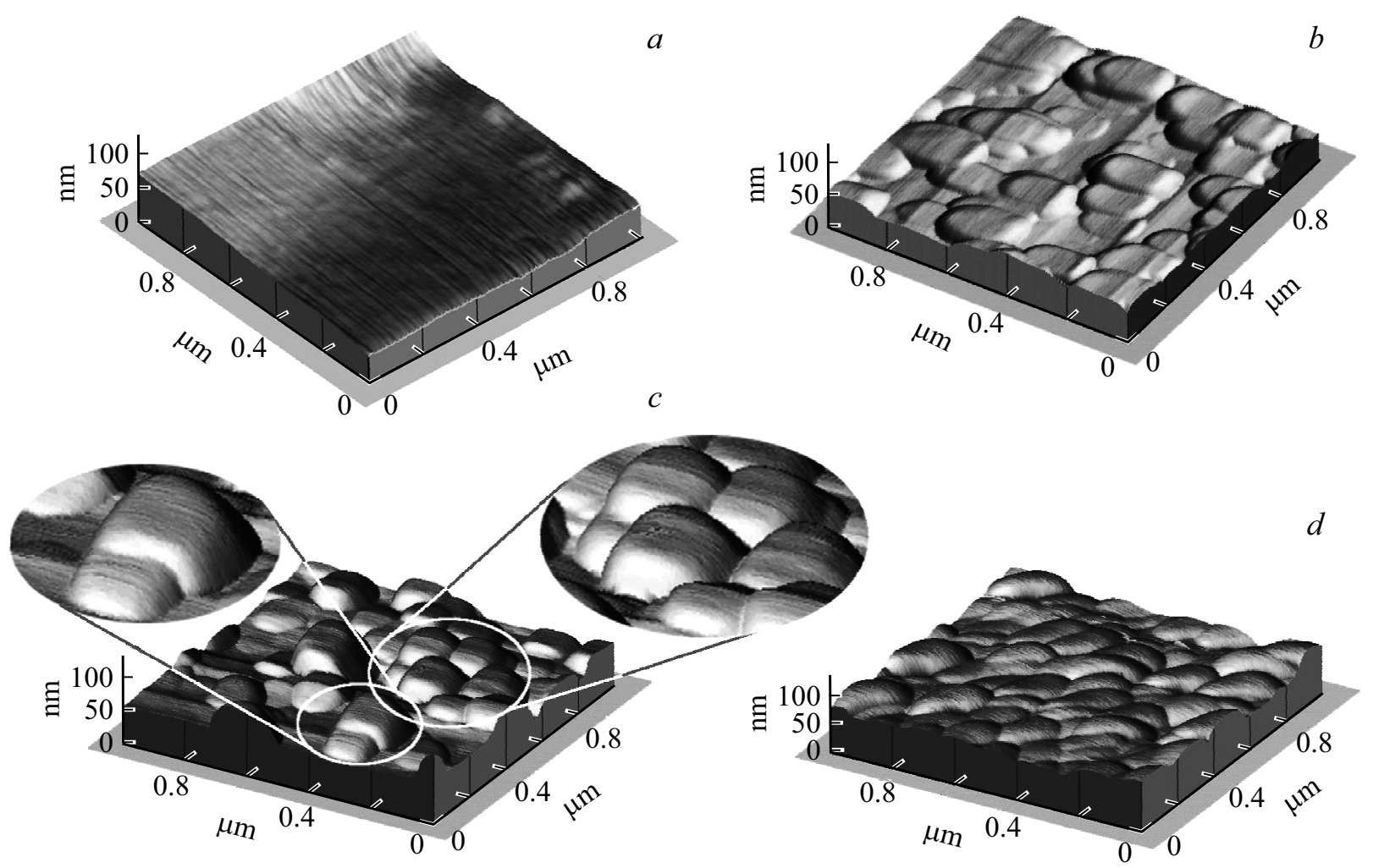

Рис. 1. Рельеф поверхности $\mathrm{Ni}_{50} \mathrm{Ti}_{50}$ в исходном состоянии $(a)$ и подвергшейся изохронным 10 min отжигам при температурах: $b-400, c-500, d-600^{\circ} \mathrm{C}$.
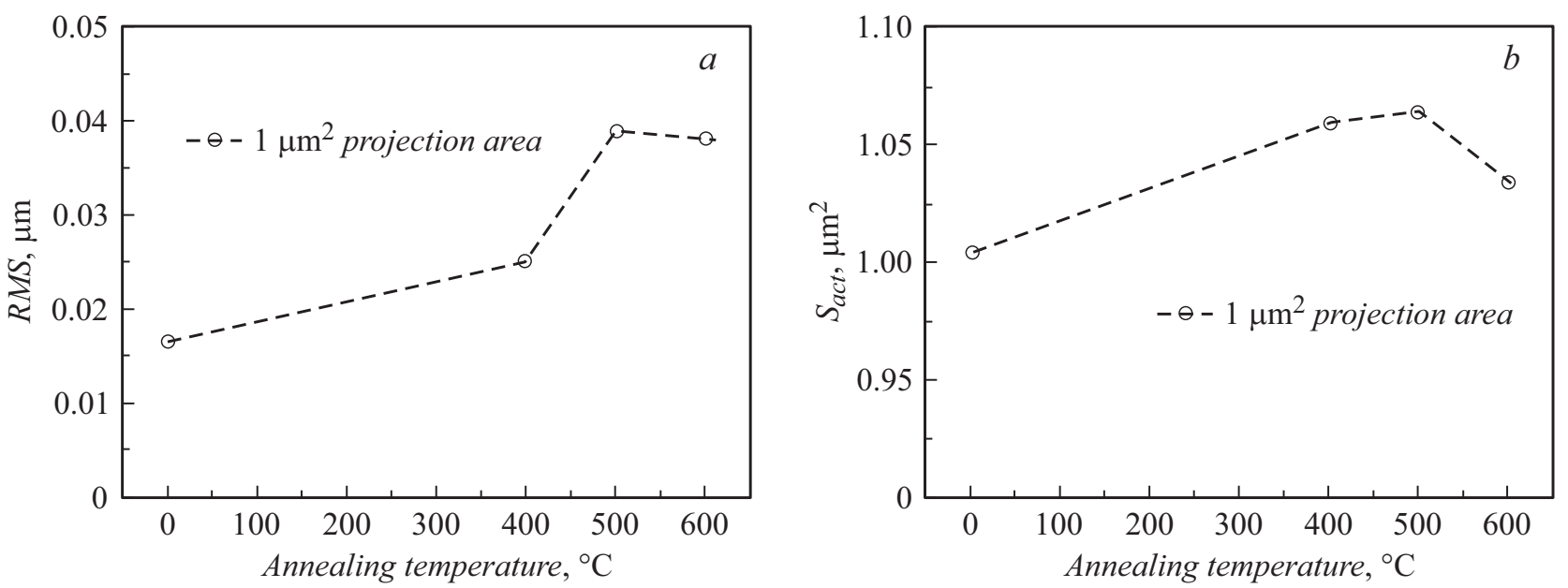

Рис. 2. Зависимость шероховатости $\left(R_{R M S}\right)(a)$ и реальной площади поверхности $\left(S_{a c t}\right)(b)$ ленты $\mathrm{Ni}_{50} \mathrm{Ti}_{50}$ от температуры изохронных отжигов.

искомый спектр $f(\alpha)$. Обе методики подробно описаны в источниках [27-30].

\section{3. Результаты и обсуждение}

Контактная сторона ленты $\mathrm{Ni}_{50} \mathrm{Ti}_{50}$ исследовалась методом РСА. В исходном состоянии были получены пики, соответствующие соединениям $\mathrm{Ti}\left(\mathrm{Ni}_{0.5} \mathrm{Cu}_{0.5}\right), \mathrm{Al}_{4} \mathrm{Ni}_{3}$,
$\mathrm{NiAl}, \mathrm{Ti}_{3} \mathrm{AlN}$, и $\mathrm{TiC}$, однако рефлексов $\mathrm{Ni}_{50} \mathrm{Ti}_{50}$ обнаружено не было. Составы, включающие $\mathrm{Cu}$ и $\mathrm{Al}$, получаются вследствие контакта расплава с бронзовым диском при формировании аморфной ленты. Металлоподобный карбид титана образуется при получении NiTi в графитовых тиглях [31]. В отожженном состоянии (все три режима) максимальную интенсивность имели рефлексы (110) и (211), что соответствует аустенитному (В2) составу $\mathrm{NiTi}$, также появились меньшие по интенсивности пики, 


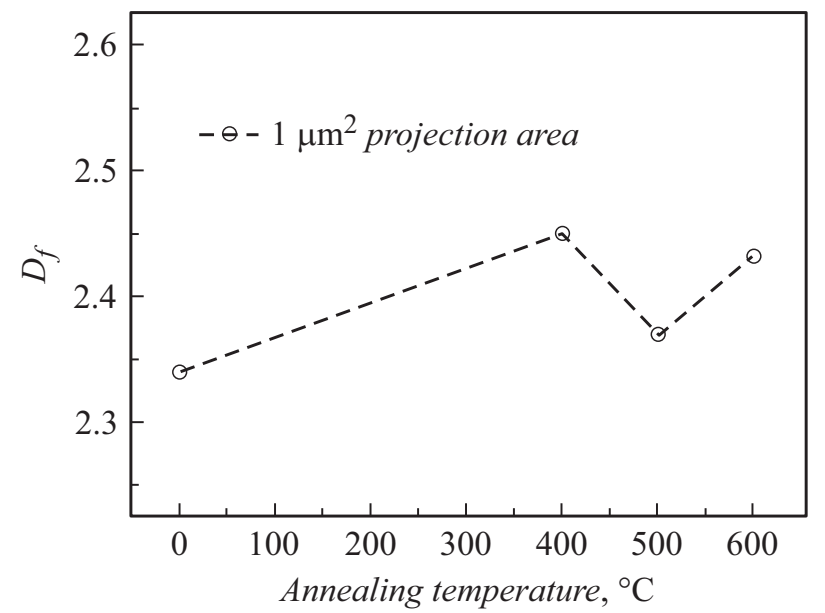

Рис. 3. Изменение $D_{f}$ поверхности $\mathrm{Ni}_{50} \mathrm{Ti}_{50}$ в зависимости от температуры изохронных отжигов.

соответствующие мартенситной $\left(\mathrm{B} 19^{\prime}\right)$ фазе NiTi (002) и (020). Помимо них наблюдались некоторые из составов, присутствующие в исходном материале, но в данном случае интенсивность этих рефлексов была очень низкая. Таким образом, поверхность ленты в исходном состоянии не являлась полностью аморфной, однако соединение $\mathrm{Ni}_{50} \mathrm{Ti}_{50}$ в кристаллическом виде появилось только после отжига.

Рельеф исходных NiTi-лент (полученный методом ACM) не имеет ярко выраженных особенностей (рис. $1, a)$, что типично для аморфного состояния вещества [8]. Тем не менее встречаются слабовыраженные неоднородности $[4,32]$, которые могут иметь кристаллическую природу и отображать особенности рельефа, унаследовавшие при изготовлении ленты [33]. Поэтому уже на данном этапе нельзя исключить присутствие зародышей кристаллизации, тем более, что информация, полученная методом РСА, это подтверждает. Результатом изохронных отжигов является появление ярко выраженных неоднородностей гранулярного типа на поверхности контактной стороны ленты $\mathrm{Ni}_{50} \mathrm{Ti}_{50}$. Гранулы на рис. $1, b$ относительно плоские, не наблюдается их агломератов. Гранулы на рис. 1, $c$ вытянуты по нормали к латеральной плоскости поверхности, они образуют скопления, кроме того, отчетливо прослеживается их „мультиярусность“. Гранулы на рис. $1, d$, как и в случае pис. $1, b$, уплощенные, однако их значительно больше, они покрывают поверхность полностью и имеют вытянутую форму. Мы считаем, что поверхности отображают различные этапы процесса роста кристаллов, более того, с подобной картиной мы сталкивались в наших предыдущих работах $[12,13]$.

На рис. 2, $a$ показано изменение параметра шероховатости $\left(R_{R M S}\right)$ контактной стороны ленты $\mathrm{Ni}_{50} \mathrm{Ti}_{50}$ в зависимости от температуры изохронных десятиминутных отжигов. Значение $R_{R M S}$ для исходной поверхности ниже, чем отожженной $(17 \mathrm{~nm}$ против максимально дости- гающихся при отжиге $500^{\circ} \mathrm{C}$ при $\left.10 \min 39 \mathrm{~nm}\right)$. Таким образом, с увеличением температуры отжига поверхностная шероховатость растет, достигая максимума при $500^{\circ} \mathrm{C}(10 \mathrm{~min})$, однако при $600^{\circ} \mathrm{C}(10 \mathrm{~min})$ ее значение падает. Подобные значения шероховатости при таких же режимах отжига получены и другими авторами $[4,34]$. По мнению авторов, повышение шероховатости происходит за счет атомной кластеризации, а последующее падение вызвано процессом миграции атомов в направлении раздела агломератов гранулярного типа - микроканавок.

Рассматривая зависимости изменения площади реальной поверхности $S_{a c t}$ (рис. $2, b$ ) контактной стороны ленты $\mathrm{Ni}_{50} \mathrm{Ti}_{50}$, отметим, что эта кривая ведет себя подобным образом (тенденция, как у $R_{R M S}$ ). Опять же наблюдается пик при температуре $500^{\circ} \mathrm{C}$. Визуально рельеф поверхностей находится в соответствии с рассчитанными выше параметрами. Действительно, уплощение рельефа поверхности количественно ведет к снижению поверхностной шероховатости, уменьшению реальной площади поверхности (рис. $1, d$, рис. $2, a, b$ ).

Как говорилось выше, важной геометрической характеристикой, дополняющей статистический анализ, является ФР поверхности. Величина $D_{f}$ показывает, по сути, меру отклонения поверхности от своей евклидовой размерности, что также связано с характеристиками $R_{R M S}$ и $S_{a c t}$. Значения $D_{f}$ были рассчитаны для исходной и отожженных поверхностей контактной стороны лены $\mathrm{Ni}_{50} \mathrm{Ti}_{50}$ (рис. 3). Величины ФР в случае отожженных образцов выше, чем в исходном состоянии, что в общем случае согласуется с возрастанием $R_{R M S}$ и $S_{a c t}$. Подобную картину мы наблюдали и при расчете методом вейвлет-преобразований (ВП) в случае поверхности МС $\mathrm{Fe}_{77} \mathrm{Ni}_{1} \mathrm{Si}_{9} \mathrm{~B}_{13}$, находящегося при механической нагрузке [35]. Зависимость $D_{f}$ при значении температуры отжига $500^{\circ} \mathrm{C}$ при $10 \mathrm{~min}$ имеет локальный минимум. Нами также было получено увеличение показателя Херста (Н) (метод ВП) при отжиге $\mathrm{MC} \mathrm{Fe}_{77} \mathrm{Ni}_{1} \mathrm{Si}_{9} \mathrm{~B}_{13}$, при появлении нанокристаллов [13]. Показатель Херста

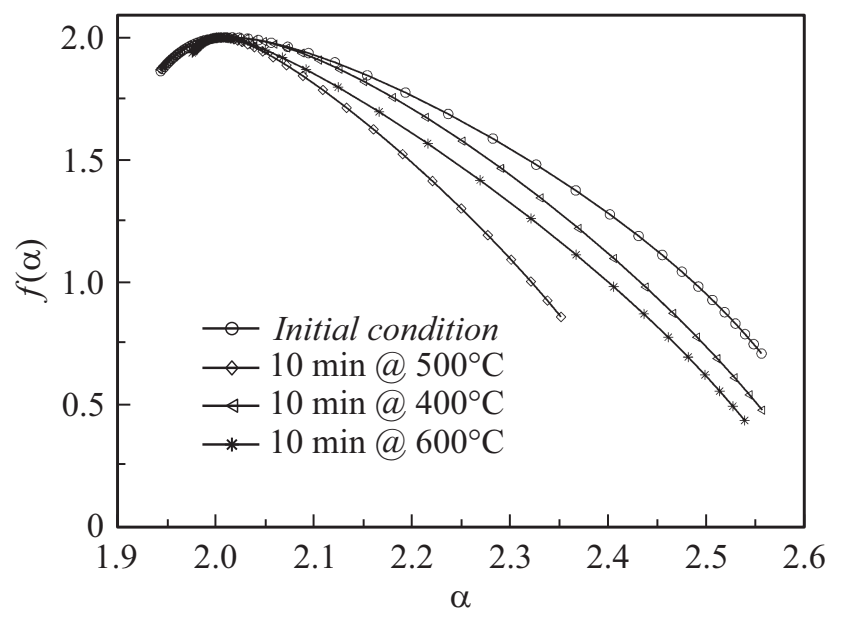

Рис. 4. Мультифрактальные спектры сингулярностей для образцов $\mathrm{Ni}_{50} \mathrm{Ti}_{50}$ в исходном и отожженном состояниях. 


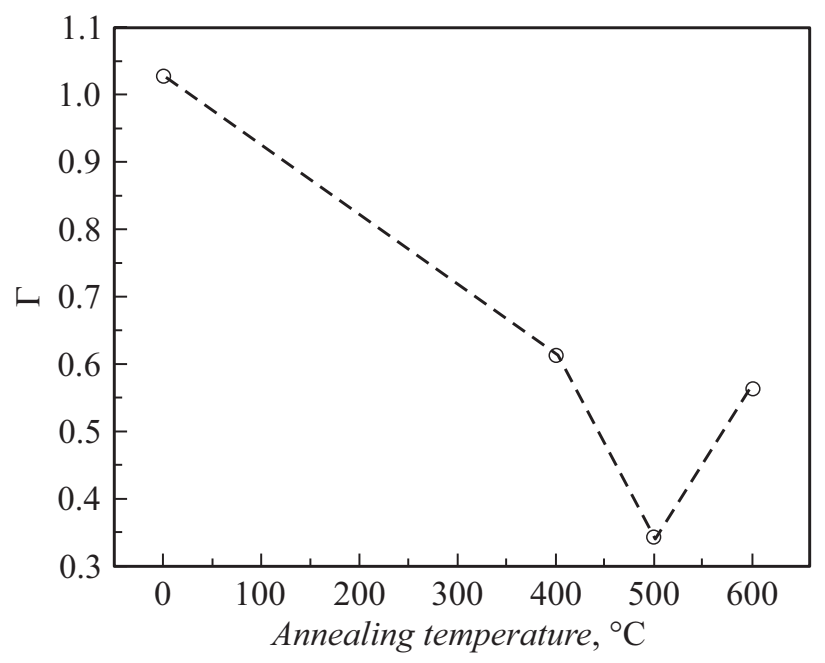

Рис. 5. Изменения ширины спектра сингулярностей в $\mathrm{Ni}_{50} \mathrm{Ti}_{50}$ в зависимости от температуры изохронных отжигов.

связан с ФР соотношением $H=E-D_{f}$, где $E-$ евклидова размерность пространства [22]. Таким образом, на наш взгляд, локальный минимум $D_{f}$ показывает, что система при данном режиме отжига может находиться в состоянии структурных перестроек.

Прибегая к расчету ФР, мы, по сути, принимаем допущение о том, что исследуемая поверхность является абсолютно однородной. Действительно, металлические стекла, являющиеся в некоторой степени гомогенными материалами (лишь топологически на определенном масштабе), могут удовлетворять этому допущению, однако для большинства поверхностей реальных материалов это не верно. Поэтому в общем случае целесообразнее применять более гибкий метод, учитывающий фактор гетерогенности поверхности материала, которым является МФ. Таким образом, были определены основные мультифрактальные характеристики для исходной и отожженных поверхностей (для проекционных площадей $1 \mu \mathrm{m}^{2}$ ) контактной стороны ленты $\mathrm{Ni}_{50} \mathrm{Ti}_{50}$. В качестве результатов получены спектры сингулярностей мультифрактала $f(\alpha)$. Для этого, согласно описанной выше методике, сначала рассчитывались статистическая сумма $Z(q, \varepsilon)$. Далее для всех моментов порядка $-5<q<5$ с шагом 0.2 в диапазоне $\varepsilon=(0 \ldots-6)$ зависимость $Z(\varepsilon)$, построенная в логарифмических координатах, оказалась линейна, следовательно, обеспечивается нахождение в области скейлинга $[27,30]$. Затем, отталкиваясь от этого, определялась скейлинговая экспонента $\tau(q)$. В свою очередь в рассматриваемом диапазоне моментов порядка $q$ эта зависимость оказалась нелинейна, из чего был сделан вывод о том, что поверхность мультифрактальна $[24,36]$. На последнем шаге определялись спектры сингулярностей $f(\alpha)$ для исходной и отожженных поверхностей (рис. 4). Все зависимости имеют ярко выраженную усеченную слева „крюкообразную“ форму, при этом для всех $\Delta f(\alpha)>0$. Исходя из этого, можно сделать заключение, что исследуемые поверхности имеют высокую степень мультифрактальности и рельеф, в котором долины преобладают над пиками [27]. Однако, на наш взгляд, причиной такого вида зависимости может являться и аппаратная ошибка метода АCM, связанная с различным качеством сканирования высот и впадин поверхности зондом [37]. Поэтому данный вопрос следует считать открытым.

Из полученных спектров сингулярностей для контактной поверхности $\mathrm{Ni}_{50} \mathrm{Ti}_{50}$ в исходном состоянии и после воздействия отжигов были рассчитаны их ширины Г (рис. 5). Ярко выраженный минимум зависимости ширины спектра сингулярностей находится при значении температуры отжига $500^{\circ} \mathrm{C}$ при $10 \mathrm{~min}$.

Как мы видим, все полученные в настоящей работе зависимости имеют экстремум при режиме термообработки $500^{\circ} \mathrm{C}$ при $10 \mathrm{~min}$. Этот факт мы связываем с наличием фазового перехода. Фазовые переходы обладают свойством скейлинга - пространственно-временной масштабной инвариантности, разновидностью которой являются фрактальные структуры. Видно, что в точке перехода наблюдается минимум ширины спектра сингулярностей $\Gamma$, что показывает переход системы к монофрактальному состоянию.

\section{Заключение}

Исходные образцы лент нитинола в целом являлись аморфными, однако было выявлено присутствие кристаллической составляющей, которая содержала упорядоченные фазы на основе Ti и Ni. Кристаллический NiTi эквиатомного состава появился лишь после десяминутного отжига при температуре $500^{\circ} \mathrm{C}$. Вместе с этим на поверхности выявлено образование агломератов неоднородностей гранулярного типа, имеющих кристаллическую природу. В зависимости от повышения температуры термообработки такие кристаллиты находятся на разных этапах своего роста.

При отжиге при температуре $500^{\circ} \mathrm{C}$ в течение $10 \mathrm{~min}$ статистические показатели геометрии поверхности имеют локальные экстремумы. В этой же точке наблюдается минимум значений ширин спектров сингулярностей, указывающий на переход системы в монофрактальное состояние. Эти события подтверждают наличие в материале фазового перехода, а именно - кристаллизации.

\section{Конфликт интересов}

Авторы заявляют, что у них нет конфликта интересов.

\section{Список литературы}

[1] Bernard W.L., Kahn H., Heuer A.H., Huff M.A. // J. Microelectromech. Syst. 1998. Vol. 7. N 2. P. 245-251.

[2] Wang X., Rein M., Vlassak J.J. // J. Appl. Phys. 2008. Vol. 103. 023501. P. 1-6. DOI: 10.1063/1.2829811 
[3] Huang X., Ramirez A.G. // Appl. Phys. Lett. 2009. Vol. 95. 121911. P. 1-3. DOI: $10.1063 / 1.3236544$

[4] Behera A., Suman R., Aich S., Mohapatra S.S. // Surf. Interfac. Anal. 2017. Vol. 49. P. 620-629. DOI: 10.1002/sia.6201

[5] Rey T., Le Cam J.B., Chagnon G., Favier D., Rebouah M. et al. // Mater. Sci. Engineer.: C. 2014. Vol. 45. P. 184-190. DOI: $10.1016 /$ j.msec.2014.08.062

[6] Kotnur V.G., Tichelaar F.D., Fu W.T., De Hosson J.T.M., Janssen G.C.A.M. // Surf. Coat. Technol. 2014. Vol. 258. N 15. P. 1145-1151. DOI: 10.1016/j.surfcoat.2014.07.018

[7] Lee H.J., Ramirez A.G. // Appl. Phys. Lett. 2004. Vol. 85. N 7. P. 1146-1148. DOI: $10.1063 / 1.1783011$

[8] Mohri M., Nili-Ahmadabadi M., Chakravadhanula V.S.K. // Mater. Characterization. 2015. Vol. 103. P. 75-80. DOI: 10.1016/j.matchar.2015.03.017

[9] Einfalt T., Planinek O., Hrovat K. // Acta Pharm. 2013. Vol. 63. P. 305-334. DOI: 102478/acph-2013-0026

[10] Hou H., Horn M.W., Hamiltona R.F. // Shape Memory and Superelasticity. 2016. Vol. 2. N 4. P. 330-336. DOI: $10.1007 / \mathrm{s} 40830-016-0093-9$

[11] Crmier L. // Procedia Mater. Sci. 2014. Vol. 7. P. 60-71. DOI: 10.1016/j.mspro.2014.10.009

[12] Betekhtin V.I., Butenko P.N., Hilarov V.L., Kadomtsev A.G., Korsukov V.E., Korsukova M.M., Obidov B.A. // Phys. Solid State. 2008. Vol. 50. N 10. P. 1875-1881. DOI: $10.1134 / \mathrm{S} 1063783408100156$

[13] Betekhtin V.I., Butenko P.N., Kadomtsev A.G., Korsukov V.E., Korsukova M.M., Obidov B.A., Tolochko O.V. // Phys. Solid State. 2007. Vol. 49. N 12. P. 2223-2229. DOI: $10.1134 / \mathrm{S} 1063783407120025$

[14] Chou H.S., Huang J.C., Chang L.W., Nieh T.G. // Appl. Phys. Lett. 2008. Vol. 93. 191901. P. 1-3. DOI: 10.1063/1.2999592

[15] Huang X., San Juan J., Ramirez A.G. // Scripta Mater. 2010. Vol. 63. P. 16-19. DOI: 10.1016/j.scriptamat.2010.02.037

[16] Nie F.L., Zheng Y.F., Cheng Y., Wei S.C., Valiev R.Z. // Mater. Lett. 2010. Vol. 64. P. 983-986. DOI: 10.1016/j.matlet.2010.01.081

[17] Ahadi A., Sun Q. // Appl. Phys. Lett. 2013. Vol. 103. 021902. P. 1-5. DOI: $10.1063 / 1.4812643$

[18] Yadav R.P., Dwivedi S., Mittal A.K., Kumar M., Pandey A.C. // Appl. Surf. Sci. 2012. Vol. 261. P. 547-553. DOI: 10.1016/j.apsusc.2012.08.053

[19] Valle F., Brucale M., Chiodini S., Bystrenova E., Albonetti C. // Micron. 2017. Vol. 100. P. 60-72.

[20] Dong L., Gao Z., Lin N. // Progr. Surf. Sci. 2016. Vol. 91. P. 101-135. 10.1016/j.progsurf.2016.08.001

[21] Korsukov V.E., Knyazev S.A., Butenko P.N. et al. // Phys. Solid State. 2017. Vol. 59. N 2. P. 308-311. DOI: $10.1134 / \mathrm{S} 1063783417020135$

[22] Mandelbrot B. The Fractal Geometry of Nature. NY: Freeman, 1982. 468 p.

[23] Feder J. Fractals. Springer Science + Business Media, LLC. 1988. 305 p.

[24] Olemskoi A.I., Flat A.Y. // Phys. Uspekhi. 1993. Vol. 163. N 12. P. 1 -50. DOI: 10.1070/PU1993v036n12ABEH002208

[25] Zahn W., Zösch A., Fresenius A. // J. Analen Chem. 1999. Vol. 365. P. 168-172. DOI: 10.1007/s002160051466

[26] Gadelmawla E.S., Koura M.M., Maksoud T.M.A., Elewa I.M., Soliman H.H. // J. Mater. Proc. Technol. 2002. Vol. 123. P. 133-145. DOI: 10.1016/S0924-0136(02)00060-2
[27] Nasehnejad M., Cholipour Shahraki M., Nabiyouni G. // Appl. Surf. Sci. 2016. Vol. 389. P. 735-741. DOI: 10.1016/j.apsusc.2016.07.134

[28] Sarkar N., Chaudhuri B. // IEEE Trans. Syst. Man Cybern. 1994. Vol. 24. P. 115-120. DOI: $10.1109 / 21.259692$

[29] Prigarin S.M., Sandau K., Kazmierczak M., Hahn K. // Intern. J. Biomathematics and Biostatistics. 2013. Vol. 2. N 2. P. $167-180$

[30] Zhang Y.H., Bai B.F., Chen J.B., Shen C.Y., Li J.Q. // Appl. Surf. Sci. 2010. Vol. 256. P. 7151-7155. DOI: $10.1016 /$ j.apssusc.2010.05.042

[31] Zhang Z., Frenzel J., Neuking K., Eggeler G. // Acta Mater. 2005. Vol. 53. P. 3971-3985. DOI: $10.1016 /$ j.actamat.2005.05.004

[32] Sharma S.K., Mohan S. // Appl. Surf. Sci. 2013. Vol. 282. P. 492-498. DOI: 10.1016/j.apsusc.2013.05.159

[33] Abd El-Rahman A.M., Wei R. // Surf. Coat. Technol. 2014. Vol. 241. P. 74-79. DOI: 10.1016/j.surfcoat.2013.08.049

[34] Mohri M., Nili-Ahmadabadi M. // Sensors \& Actuators: A. Phys. 2015. Vol. 228. P. 151-158.

[35] Hilarov V.L., Korsukov V.E., Butenko P.N., Svetlov V.N. // Phys. Solid State. 2004. Vol. 46. N 10. P. 1868-1872. DOI: $10.1134 / 1.1809422$

[36] Pavlov A.N., Anishchenko V.S. // Phys. Uspekhi. 2007. Vol. 50. N 8. P. $819-834$. DOI: $10.1070 / \mathrm{PU} 2007 \mathrm{v} 050 \mathrm{n} 08 \mathrm{ABEH} 006116$

[37] Корсуков В.Е., Анкудинов А.В., Бетехтин В.И., Бутенко П.Н., Вербиикий В.Н., Гиляров В.Л., Корсукова М.М., Нарыкова М.В., Обидов Б.А. // ФТТ. 2019. Т. 61 Вып. 4. C. 708-714. DOI: $10.21883 /$ FTT.2019.04.47417.322 